\title{
Ganglion Cyst in the Supraspinous Fossa: Arthroscopically Undetectable Cases
}

\author{
HISAO SHIMOKOBE, MASAFUMI GOTOH, YASUHIRO MITSUI, EIICHIRO YOSHIKAWA, \\ SHINICHIRO KUME, TAKAHIRO OKAWA, FUJIO HIGUCHI, \\ KENSEI NAGATA* AND NAOTO SHIBA*
}

Department of Orthopedic Surgery, Kurume University Medical Center, Kurume 839-0863, and

*Department of Orthopedic Surgery, Kurume University School of Medicine,

Kurume 830-0011, Japan

Received 4 September 2012, accepted 28 January 2013

J-STAGE advance publication 7 August 2013

Edited by KEIICHIRO NAKAMURA

\begin{abstract}
Summary: Studies have demonstrated favorable outcomes of arthroscopic decompression for ganglion cyst in the supraspinous fossa; however, little attention has been paid to the difficulty in detecting these cysts during arthroscopy. In this report, we present 2 cases in which ganglion cysts in the supraspinous fossa were undetectable during arthroscopy. The ganglion cysts were not identified in these cases during surgery despite arthroscopic decompression being performed through the area in which the cyst was expected until the suprascapular nerve was entirely exposed. After surgery, magnetic resonance imaging (MRI) confirmed the disappearance of the ganglion cyst and external rotation strength was fully improved, without shoulder pain. We emphasize here that surgeons should be aware of this difficulty when performing arthroscopic decompression of ganglion cysts in the supraspinous fossa.
\end{abstract}

Key words supraspinous fossa, ganglion cyst, arthroscopy

\section{INTRODUCTION}

Ganglion cysts in the supraspinous fossa can cause shoulder pain or lead to atrophy of the supraspinatus or infraspinatus muscle by compressing the suprascapular nerve. Various surgical procedures such as open resection [1], computed tomography/ultrasoundguided aspiration [2], or arthroscopic decompression [3] have been described. In recent years, several studies have reported favorable outcomes of arthroscopic decompression in treating suprascapular nerve palsy caused by ganglion cysts in the supraspinous fossa [3-8]. However, Moore et al. [9] reported difficulty identifying the ganglion in 6 of 11 patients during arthroscopic decompression of ganglion cysts. In this study, we report 2 cases in which the ganglion cysts in the supraspinous fossa were undetectable in arthroscopic surgery.

\section{CASE REPORTS}

\section{Case 1}

A 45-year-old man experienced dull pain in the right shoulder particularly during overhead activity with no apparent cause for 2 years. The patient visited a nearby hospital, where magnetic resonance imaging (MRI) found a ganglion cyst in the supraspinous fossa. The patient was then referred to our hospital.

The patient had normal range of motion in the right shoulder joint. The impingement signs of Neer [10] and Hawkins [11] tested negative. Atrophy of the right infraspinatus muscle was observed. A manual

Correspondence to: Masafumi Gotoh, MD, PhD, Department of Orthopedic Surgery, Kurume University Medical Center 155 Kokubu-machi, Kurume, Fukuoka 839-0863, Japan. Tel.: +81-942-22-6111 ex. 533; Fax: +81-942-22-6657 E-mail: gomasa@med.kurume-u.ac.jp 
muscle test (MMT) showed decreased external rotation strength (MMT score, 4). Plain radiography found no apparent abnormalities. A T2-weighted MRI of the right shoulder found a multilobular ganglion cyst in the suprascapular fossa (Fig. 1).

Surgery was performed under general anesthesia with the patient in the beach chair position. A posterior viewing portal and an anterior working portal were

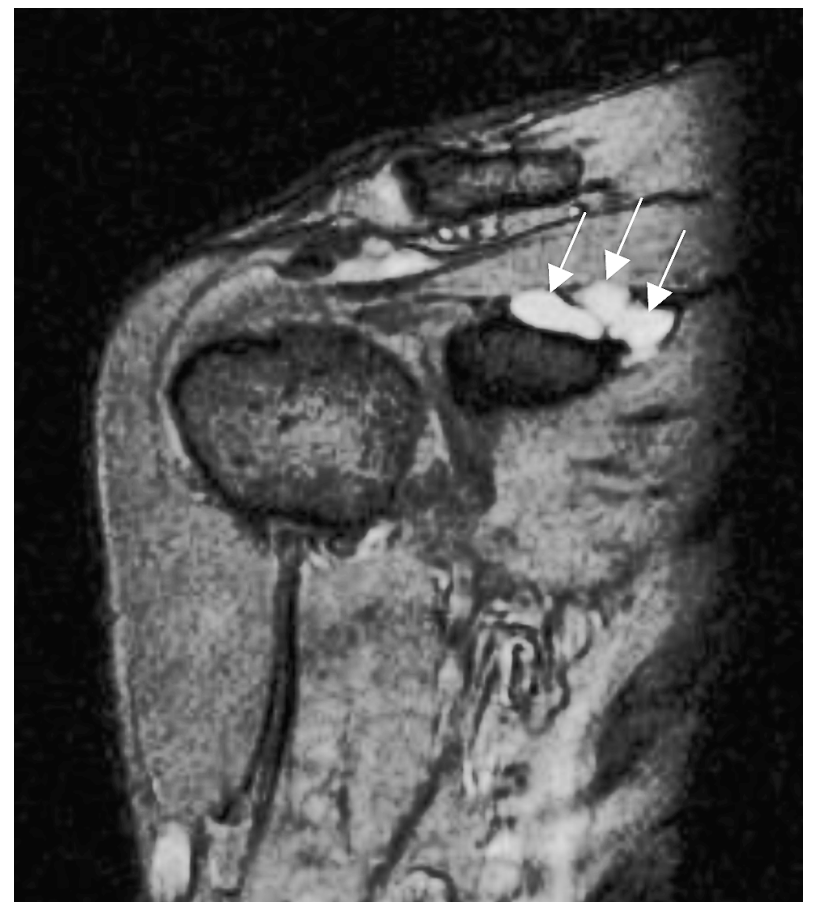

Fig. 1. Case 1: T2-weighted magnetic resonance imaging of the right shoulder 2 months before the surgery. White arrows: ganglion cysts.

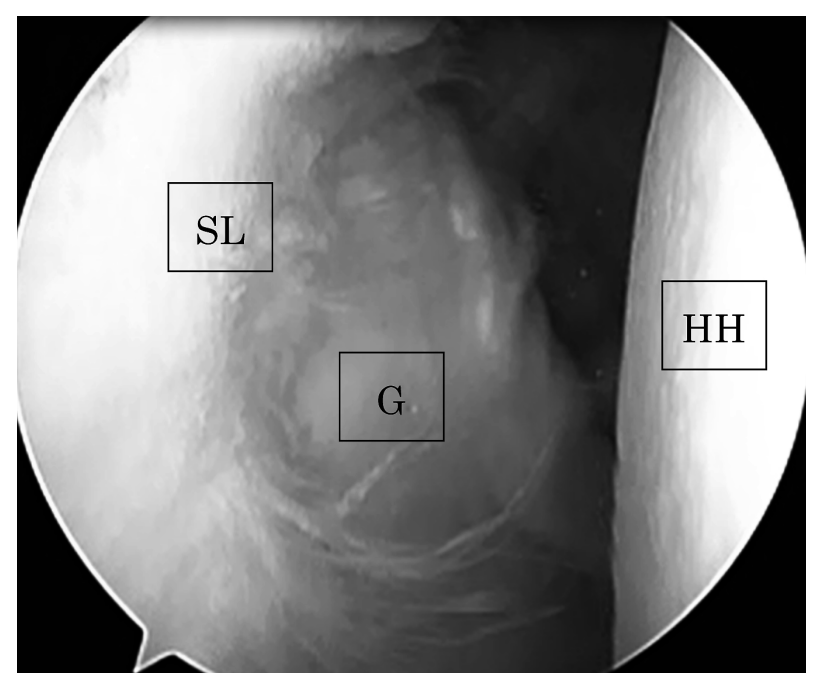

Fig. 2. Case 1: arthroscopic findings showing the superior labral lesion.

SL: Superior labrum, G: Glenoid, HH: Humeral head. created. The intra-articular arthroscopy showed a lesion of the superior glenoid labrum from the 10 o' clock to 2 o'clock positions (Fig. 2). No rotator cuff tear of the long head tendon of the biceps lesion or cartilaginous damage of the humeral head or glenoid was detected. An incision was made into the capsule beneath the damaged labrum, and a thorough exploration was performed to prevent injury to the suprascapular nerve. Despite these measures, ganglion cysts were not identified. Subacromial bursoscopy also failed to identify the cyst in the supraspinous fossa. Arthroscopic decompression was performed using blunt instruments and a motorized shaver in the area determined by the preoperative MRI. Labral repair was performed using a bioabsorbable suture anchor (Depuy, USA). After a suture hook (Linvatec, USA) penetrated the superior labrum at its capsular reflection, the labrum was repaired by attachment to the anchor with a sliding knot and 3 alternating half-hitches.

The ganglion was no longer visible on an MRI taken 3 months postoperatively. Two years after the surgery, the patient was free of pain without range of motion limitations in his right shoulder. The external rotation strength and atrophy of the infraspinatus muscle were completely improved. He has returned to work as a manual laborer.

\section{Case 2}

A 36-year-old man experienced dull pain in his right shoulder during overhead activity and weakness during external rotation with no particular cause for 1 year. Conservative therapy with a nonsteroidal antiinflammatory analgesic failed to relieve his symptoms. The patient was then referred to our hospital.

He had normal range of motion in the right shoulder. The impingement signs of Neer [10] and Hawkins [11] tested negative. Atrophy of the right infraspinatus muscle was observed. An MMT score of 4 indicated decreased external rotation strength. Plain radiography found no apparent abnormalities. A T2-weighted MRI on the right shoulder found a multilobular ganglion cyst on the suprascapular notch (Fig. 3).

Surgery was performed under general anesthesia with the patient in the beach chair position. Arthroscopy showed a lesion of the posterior superior glenoid labrum. No other abnormalities were found in the glenohumeral joint. As in case 1, arthroscopic exploration found no ganglion. Therefore, arthroscopic decompression was performed using blunt instruments and a motorized shaver in the area determined by the preoperative MRI. Labral repair was performed using a bioabsorbable suture anchor (Depuy, USA). 


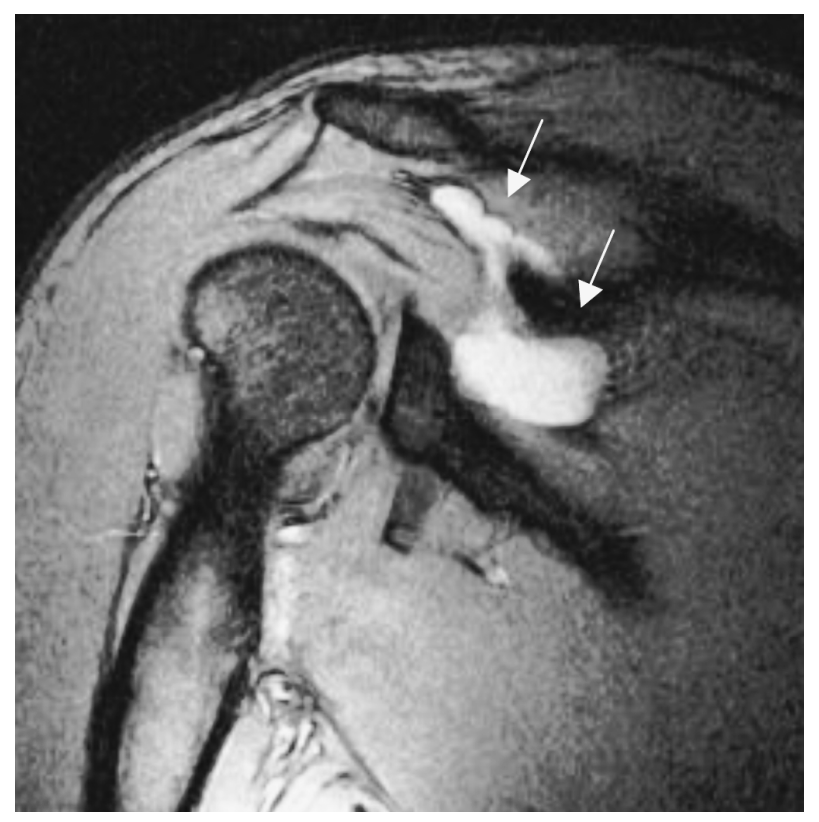

Fig. 3. Case 2: T2-weighted magnetic resonance imaging of the right shoulder 4 days before the surgery. White arrows: ganglion cysts.

The ganglion was no longer visible on MRI taken 3 months postoperatively. At 18 months after the surgery, the patient's right shoulder pain has ameliorated, range of motion is not limited, and the external rotation strength and atrophy of the infraspinatus muscle have also improved. The patient now leads an unhindered daily life and has returned to work as a manual laborer.

\section{DISCUSSION}

Ganglion cysts in the supraspinous fossa have previously been thought to be caused by synovial fluid leakage due to 1-way mechanisms or by mucous changes in the tissues around the capsulolabral complex $[6,12,13]$. In our patients, who worked as manual laborers, minor trauma or repetitive overuse may have been the cause of capsulolabral complex injury. Several studies have reported favorable outcomes after arthroscopic decompression of ganglion cysts in the supraspinous fossa [3-8]. However, few cases in which the ganglion was difficult to identify during arthroscopy have been reported; in fact, to the best of our knowledge, only Moore et al. [9] have reported such a case.

We previously experienced 4 cases of ganglion cyst in the supraspinous fossa in which arthroscopic decompression was undertaken. In these cases, the ganglion cyst was undetectable in 2 of 4 cases. This finding was similar to that reported by Moore et al. [9] (in
6/11 patients), who described the difficulty in arthroscopic detection of the ganglion cyst in the supraspinous fossa.

In the cases reported here, as the ganglion cyst was undetectable during arthroscopy, on the basis of the preoperative MRI data we decompressed the inferred site of the ganglion cyst until the suprascapular nerve was entirely decompressed. The reasons why the ganglion cysts were undetectable during the arthroscopy were not determined in this report, but we suggest the following possibilities: (1) the ganglion cyst was aspirated with the shaver without being noticed; (2) the ganglion cyst flowed out along with the circulating fluid at some unknown point. We suggest that whenever this procedure is performed, it will be important to maintain careful intraoperative observation, and to recognize that in some cases the ganglion may be difficult to identify during surgery.

Previous studies reported that labral repair alone led to cyst resolution and pain relief without cyst decompression [14,15]. Youm et al. [14] reported that repair of a labral tear, without cyst excision, resulted in successful outcomes for all ten patients who had spinoglenoid cysts associated with superior labral tears. Schroder et al. [15] also reported that most ganglion cyst in the supraspinous fossa resolve, and patient satisfaction can be expected to be high after labral repair alone without cyst decompression. These results suggest that even if the ganglion cysts in the supraspinous fossa were undetectable as in the present case, labral repair alone might lead to good clinical outcomes. Future studies involving a larger number of subjects and longer follow-up periods are needed to confirm this possibility.

We have experienced 2 cases in which the ganglion cyst was undetectable during arthroscopy but resulted in obtained favorable outcomes without recurrence by decompression of the inferred site until the suprascapular nerve was completely exposed. Previous reports have demonstrated acceptable outcomes of arthroscopic decompression of the cyst in the supraspinous fossa [3-8]; however, little attention has been paid to the difficulty in detecting such cysts during arthroscopy. Similar cases may be more common than generally believed. We therefore emphasize that surgeons should be aware of this difficulty when performing arthroscopic decompression of the ganglion cyst in the supraspinous fossa.

\section{REFERENCES}

1. Hirayama T and Takemitsu Y. Compression of the supras- 
capular nerve by a ganglion at the suprascapular notch. Clin Orthop Relat Res 1981; 155:95-96.

2. Leitschuh PH, Bone CM, and Bouska WM. Magnetic resonance imaging diagnosis, sonographically directed percutaneous aspiration, and arthroscopic treatment of a painful shoulder ganglion cyst associated with a SLAP lesion. Arthroscopy 1999; 15:85-87.

3. Iannotti JP and Ramsey ML. Arthroscopic decompression of a ganglion cyst causing suprascapular nerve compression. Arthroscopy 1996; 12:739-745.

4. Bigliani LU, Dalsey RM, McCann PD, and April EW. An anatomical study of the suprascapular nerve. Arthroscopy 1990; 6:301-305.

5. Ghodadra N, Nho SJ, Verma NN, Reiff S, Piasecki DP et al. Arthroscopic decompression of the suprascapular nerve at the spinoglenoid notch and suprascapular notch through the subacromial space. Arthroscopy 2009; 25:439-445.

6. Kessler MA, Stoffel K, Oswald A, Stutz G, and Gaechter A. The SLAP lesion as a reason for glenolabral cysts: a report of five cases and review of the literature. Arch Orthop Trauma Surg 2007; 127:287-292.

7. Lichtenberg S, Magosch P, and Habermeyer P. Compression of the suprascapular nerve by a ganglion cyst of the spinoglenoid notch: the arthroscopic solution. Knee Surg Sports Traumatol Arthrosc 2004; 12:72-77.

8. Westerheide KJ, Dopirak RM, Karzel RP, and Snyder SJ.
Suprascapular nerve palsy secondary to spinoglenoid cysts: results of arthroscopic treatment. Arthroscopy 2006; 22:721-727.

9. Moore TP, Fritts HM, Quick DC, and Buss DD. Suprascapular nerve entrapment caused by supraglenoid cyst compression. J Shoulder Elbow Surg 1997; 6:455-462.

10. Neer CS 2nd and Welsh RP. The shoulder in sports. Orthop Clin North Am 197; 8:583-591.

11. Hawkins RJ and Kennedy JC. Impingement syndrome in athletes. Am J Sports Med 1980; 8:151-158.

12. Tirman PF, Feller JF, Janzen DL, Peterfy CG, and Bergman AG. Association of glenoid labral cysts with labral tears and glenohumeral instability: radiologic findings and clinical significance. Radiology 1994; 190:653-658.

13. Nakama K, Gotoh M, Mitsui Y, Shirachi I, Higuchi F et al. Use of autologous fibrin sealants to treat ganglion cysts: a report of two cases. J Orthop Surg (Hong Kong) 2010; 18:104-106.

14. Youm T, Matthews PV, and El Attrache NS. Treatment of patients with spinoglenoid cysts associated with superior labral tears without cyst aspiration, debridement, or excision. Arthroscopy 2006; 22:548-552.

15. Schroder CP, Skare O, Stiris M, Gjengedal E, Uppheim G et al. Treatment of labral tears with associated spinoglenoid cysts without cyst decompression. J Bone Joint Surg Am 2008; 90:523-530. 